\title{
The SAR Angle: A Contemorary Sagital Jaw Dussplasia Marker
}

\author{
Dr Sonahita Agarwal,' Dr Jitendra Bhagchandani,2 Dr Praveen Mehrotra,3 \\ Dr Sudhir Kapoor, ${ }^{4}$ Dr Raj Kumar Jaiswals \\ 2Senior Lecturer, 34.4Professor, sReader \\ Dept of Orthodontics, Sardar Patel PG Institute of Dental Sciences, Lucknow, India \\ Correspondence: Dr Sonahita Agarwal; email: sonasand2ooo@yahoo.com
}

\section{ABSTRACT}

Introduction: Cephalometric analysis forms the backbone of orthodontic diagnosis and treatment planning. However most of the angles used to assess antero-posterior jaw base discrepancy are based on landmarks that change with age, jaw rotation and orthodontic treatment. Walkers point is one landmark that has been suggested to be stable and easy to locate on radiograph.

Objective: To derive SAR angle based on Walkers point, points $M$ and $G$ to assess true sagittal discrepancy.

Materials \& method: Sixty pretreatment lateral cephalograms of North Indian subjects were grouped in three classes of skeletal pattern based on fulfillment of any two of the three criteria: ANB angle, Witt's appraisal and Beta angle. The mean and SD for the SAR angle in three groups were calculated. ANOVA one-way of variance and Newman-Keuls tests were done to compare the groups.

Result: The mean value for SAR angle of Class I skeletal pattern group was $55.98^{\circ}$ (SD 2.24), whereas mean value for Class II and Class III skeletal groups were $50.18^{\circ}$ and $63.65^{\circ}$ with standard deviations 2.70 and 2.25 respectively. The receiver operating characteristic curves show that the cutoff point between Class I and Class II groups could be considered a SAR angle of approximately $53^{\circ}$, and the cutoff point between Class I and Class III groups could be considered a SAR angle of approximately $59^{\circ}$.

Conclusion: The SAR angle can be a reliable diagnostic aid to assess the sagittal jaw discrepancy more consistently.

Keywords: anteroposterior discrepancy, cephalometric, diagnostic aid, sagittal discrepancy

\section{INTRODUCTION}

Antero-posterior jaw discrepancies form an important part of orthodontic diagnosis and treatment planning. Various cephalometric measurements have been derived to assess the sagittal discrepancies. However most of these parameters are based on either direct linear or angular measurements. The problem that was commonly faced using these parameters was the variability of the landmarks with jaw rotation, head posture, orthodontic treatment as well as growth.'

The most commonly used angle ANB, developed by Riedel ${ }^{2}$ has been proved to be unreliable indicator of apical base discrepancies. ${ }^{3,4}$ The stability of nasion has been in question as it does not remain stable with age. Also, the rotation of jaws as a result of growth or orthodontic treatment or even the rotation of head during the shooting of lateral cephalogram has a direct influence on ANB angle reading. 5,6,7

Another popular measurement that was suggested to be an answer to the shortcomings of ANB was the Wits appraisal developed by Jacobson; ${ }^{3}$ although the use of functional occlusal plane overcame the problems faced with ANB, it created many problems of its own. Any change in the angulation of functional occlusal plane caused profound change in the measurement reading. The difficulty in identification of the occlusal plane especially in patients in mixed dentition stage, open bite, skeletal asymmetries, missing teeth or multiple impactions also posed additional problem. ${ }^{8.9,10}$

Beta angle;" derived by Baik and Ververidou in 2004, assessed true apical base relationship independent of cranial reference plane or occlusal plane. Although it gave a reliable picture of the sagittal discrepancy, it still used points A and $B$ which were unstable landmarks as they were subject to alveolar bone remodeling with the change in position of incisors. ${ }^{12,13}$ The identification of condylion and point A was not easily reproducible on lateral cephalograms..$^{14,15}$ 
Recently two more angles, Yen angle ${ }^{16}$ and $\mathrm{W}$ angle ${ }^{17}$ were derived to address the shortcomings of beta angle. Yen angle is vulnerable to changes with the rotation of jaws that could be either due to growth or orthodontic treatment. Although $W$ angle is not affected by the rotation of jaws, it depends on Point $S$, the midpoint of Sella turcica which is again an unstable landmark as proved by many studies. 18,19,20

The purpose of this study was to develop an angle based on stable landmarks that would measure the true apical base discrepancy and would not be susceptible to changes in jaw rotation or growth occurring during orthodontic treatment.

\section{THE SAR ANGLE}

The SAR angle is a new parameter for assessing the sagittal apical base discrepancy. It uses the three skeletal reference points:

Point M: Midpoint of the premaxilla

Point G: Center of the largest circle that is tangent to the internal inferior, anterior, and posterior surfaces of the mandibular symphysis. ${ }^{21}$

The centre of premaxilla and mandibular symphysis were identified by constructing a template with concentric circles whose diameters increased in 0.5 inch increments. Center of the template was marked, and Point $M$ and Point $G$ were identified on the tracings.

Point W (Walkers Point): The mean intersection point of the lower contours of the anterior clinoid processes and the contour of the anterior wall of sella turcica.

The three lines that would form joining these points are:

- L Line connecting Point $M$ and Point $G$

- Line connecting Point W and Point $G$

- Line from point M perpendicular to the W-G line

The angle that would be measured will be between the perpendicular line from point $M$ to $W-G$ line and the $M-G$ line is the SAR angle (Figure 1).

The aim of this study was to define the mean values and standard deviation for this angle in subjects with Class I, Class II and Class III skeletal patterns.

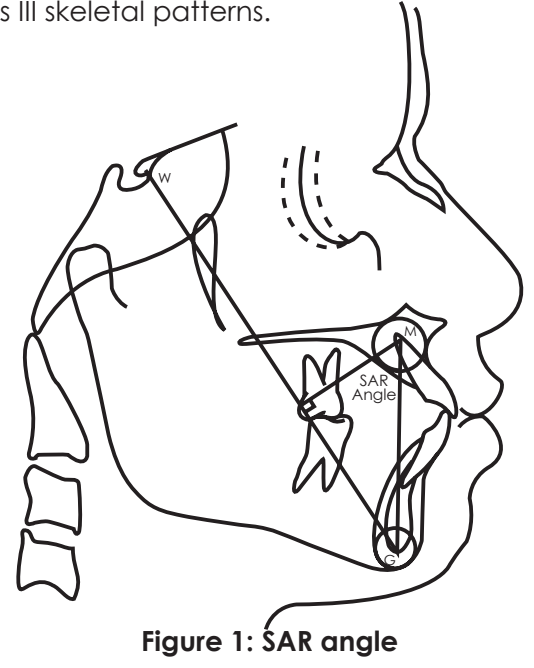

\section{MATERIALS AND METHOD}

The sample consisted of 60 pretreatment lateral cephalograms of 13-25 years old individuals from the orthodontic files of Department of Orthodontics, Sardar Patel Postgraduate Institute of Dental \& Medical Sciences. These cephalograms were traced and ANB angle, Wits appraisal and beta angle were measured to assign the lateral cephalograms to Class I, II and III groups. To be included in either group; samples were required to have a minimum of two parameters indicating Class I, II or III skeletal relationship.

After the initial tracing, ten random lateral cephalograms were retraced by each operator separately at a week interval and the mean of those values were calculated. The combined error was calculated with the Dahlberg's formula. The mean difference was within $0.5^{\circ}$ for the measurements and was insignificant.

For patients to be in Class I skeletal pattern group, a minimum of two of the following three criteria should be fulfilled: ANB of 2-4 $4^{\circ}$, Wits coincidence of $A O$ and $B O$ in females or $B O 1$ $\mathrm{mm}$ ahead of $A O$ in males and a Beta angle of 27-35. 20 lateral cephalograms (10 males, 10 females) met the above mentioned criteria.

Similarly, Class II skeletal pattern group was indicated by an ANB greater than $4^{\circ}$, Wits appraisal with $A O$ ahead of $B O$ in females or $A O$ coinciding with or ahead of $B O$ in males and Beta angle of less than $27^{\circ}$. Again 20 lateral cephalograms (10 males, 10 females) comprised the skeletal Class II group.

Class III skeletal pattern group was indicated by an ANB less than $2^{\circ}$, Wits $B O$ ahead of $A O$ in females or $B O$ ahead of $A O$ by more than $1 \mathrm{~mm}$ in males and Beta angle of more than $35^{\circ}$. 20 lateral cephalograms ( 10 males, 10 females) met the above criteria to be included in the skeletal Class III group.

The data were summarized as mean \pm SD. Groups were compared by factoral analysis (Gender and Class) of variance (ANOVA) and the significance of mean difference within and between the groups was done by Newman-Keuls post hoc test. Groups were also compared by one factor (Class) ANOVA followed by Newman-Keuls post hoc test. Receiver operating characteristics (ROC) curves analysis was done to examine the sensitivity and specificity of SAR angle as a test to discriminate between the three different skeletal pattern groups. A p-value $\leq 0.05$ was considered to be statistically significant. All statistics were performed in SPSS Version 14.

\section{RESULT}

The SAR angle of two gender and three class groups are summarized in Table 1. The mean value for the SAR angle of Class I skeletal pattern group was $55.98^{\circ}$ (SD 2.24), whereas mean value for Class II and Class III skeletal group were $50.18^{\circ}$ and $63.65^{\circ}$ with a standard deviations of 2.70 and 2.25 respectively.

The one way ANOVA revealed similar SAR angle between the two genders which was non-significant ( $F=0.07, p=0.795$ ) (Figure 2). 
Further, Newman-Keuls test revealed that the mean SAR angle of Class III was significantly different $(p<0.001)$ and higher as compared to both Class I and Class II while the mean SAR angle of Class II lowered significantly $(p<0.001)$ as compared to Class I (Table 2, Figure 3).

The sensitivity and specificity of SAR angle between Class I and Class II, and Class I and Class III are summarized in Table 3. Reciever operating characteristic curves showed that a SAR angle of less than $53^{\circ}$ had $100 \%$ sensitivity and $85 \%$ specificity for discriminating a Class II case from a Class I case. A SAR angle of more than and equal to $59^{\circ}$ had a sensitivity of $100 \%$ and specificity of $100 \%$ to discriminate a Class III case from a Class I case. Therefore the receiver operating characteristics curves show that the cutoff point between the Class I and Class II groups could be considered a SAR angle of approximately $53^{\circ}$, and the cutoff point between the Class I and Class III groups could be considered a SAR angle of approximately $59^{\circ}$.

Therefore, it can be predicted with a high degree of certainty that a patient with a SAR angle between $53^{\circ}$ and $59^{\circ}$ truly has a Class I skeletal pattern. The results also indicate that a patient with a SAR angle less than $53^{\circ}$ can be considered to have a Class II skeletal pattern, and one with a SAR angle greater than $59^{\circ}$ will most likely have a Class III skeletal pattern.

Table 1: Gender-wise descriptive statistics of SAR angle of three skeletal groups

\begin{tabular}{|l|c|c|c|}
\hline Gender & Class I & Class II & Class III \\
\hline Male $(n=10)$ & $56.10 \pm 2.60$ & $50.35 \pm 2.11$ & $63.60 \pm 2.41$ \\
\hline Female $(n=10)$ & $55.85 \pm 1.94$ & $50.00 \pm 3.30$ & $63.70 \pm 2.21$ \\
\hline Total $(n=20)$ & $55.98 \pm 2.24$ & $50.18 \pm 2.70$ & $63.65 \pm 2.25$ \\
\hline
\end{tabular}

Table 2: Student-Newman-Keuls test for pair-wise comparisons of group mean

\begin{tabular}{|c|c|c|c|c|}
\hline \multirow{2}{*}{ Group } & \multirow{2}{*}{$\mathbf{N}$} & \multicolumn{3}{|c|}{ Subset } \\
\hline & & 1 & 2 & 3 \\
\hline$\|$ & 20 & 50.18 & - & - \\
\hline I & 20 & - & 55.98 & - \\
\hline III & 20 & - & - & 63.65 \\
\hline Significance & & 1.000 & 1.000 & 1.000 \\
\hline
\end{tabular}

Table 3: Sensitivity and specificity of SAR angle

\begin{tabular}{|c|c|c|c|c|c|c|}
\hline Class & Cutoff value & Sensitivity $(\mathbf{9 5 \%} \mathbf{~ C l})$ & Specificity $(\mathbf{9 5 \%} \mathbf{C l})$ & AUC & $\mathbf{+ P V}$ & $\mathbf{- P V}$ \\
\hline Class I vs. Class II & $<53$ & $100.00(83.0-100.0)$ & $85.00(62.1-96.6)$ & $0.989 * * *$ & 87.0 & 100.0 \\
\hline Class I vs. Class III & $>59.0$ & $100.00(83.0-100.0)$ & $100.00(83.0-100.0)$ & $1.000^{* * *}$ & 100.0 & 100.0 \\
\hline
\end{tabular}

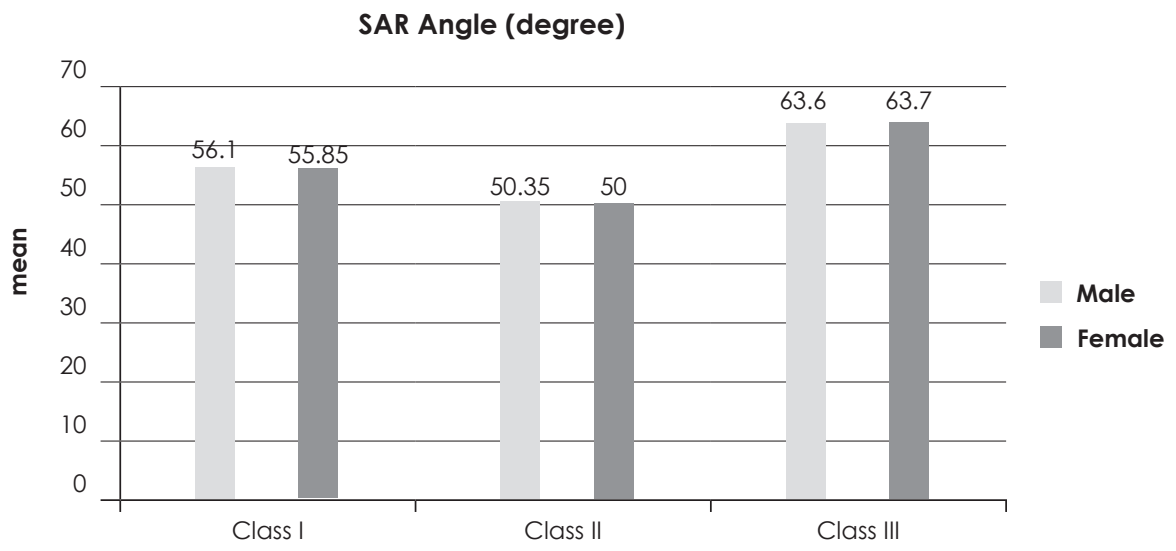

Figure 2: Mean SAR angle of three skeletal Classes in males and females 
SAR Angle (degree)

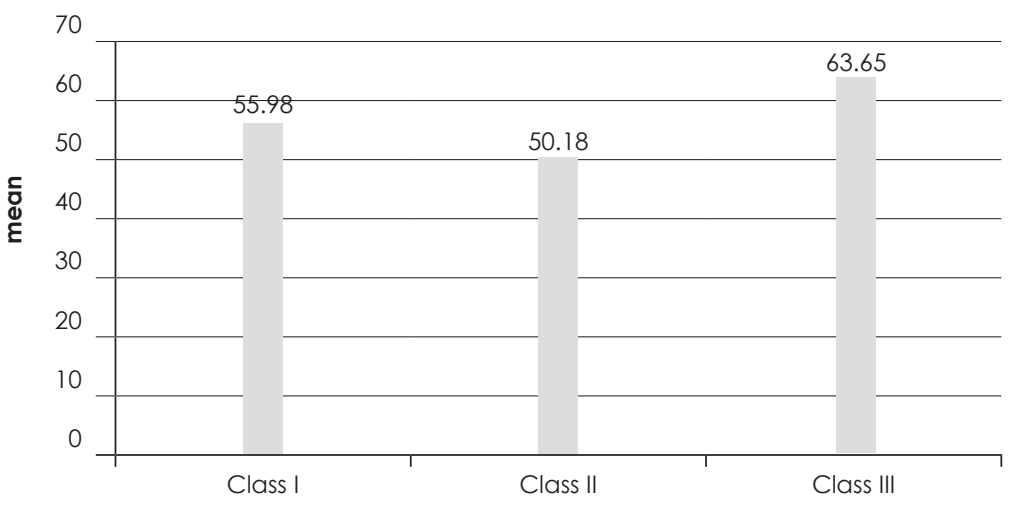

Figure 3: Mean value of the SAR angle in the three skeletal groups.

\section{DISCUSSION}

Almost all methods developed to determine the anteroposterior skeletal base relationship have some inherent limitations. The most popular measurement ANB angle is influenced by variation in the position of nasion and rotational jaw effects. Similarly Wits appraisal uses the functional occlusal plane, a dental parameter which is again subject to variation with growth or orthodontic treatment.

Although recently introduced angles such as beta angle, yen angle and $w$ angle have managed to overcome some of the existing limitations, certain demerits still prevail which questions their reliability. Ideally, an angle used to denote sagittal jaw base discrepancy should be based on stable landmarks, easy to locate and reproducible.

The development of SAR angle is a step forward in this direction. Points $M$ and point $G$ have already been proved to be an advantage over locating points $A$ and $B$ as which are susceptible to remodeling changes especially during orthodontic treatment.

Eccentric remodeling of the sella turcica during the whole growth period results in a displacement of point sella often in a backward-downward direction. Sella therefore can not be used as a stable reference point. 18,19,20 Longitudinal growth studies by Björk ${ }^{18}$ using metallic bone markers in children have shown that certain structures in the anterior cranial base are stable after a certain age. These observations have been verified histologically by Melsen. ${ }^{20}$ One of these structures, the Walkers point was found to be stable after the age of five. ${ }^{18-20,22-24}$ Arat et $\left.a\right|^{25}$ also found that the length of the mid-cranial base (W-SE) remains unchanged in all periods of pubertal growth. A recent study done by Lino et $a^{26}$ to evaluate the age related changes in the soft tissue profile from second to fourth decade used the Walkers point as a stable reference point for their analysis.

The SAR angle can be a valuable tool in orthodontic diagnosis and treatment planning as it is not influenced by growth, jaw rotations, orthodontic treatment or any other factor previously associated with other angles. Since it is independent of the anterior cranial base length, it reflects true sagittal discrepancy of the jaw bases.

The SAR angle is a step forward in deriving cephalometric analysis based on stable landmarks. However, a clinician must be aware of the importance of other cephalometric measurements in orthodontic treatment planning. The SAR angle is an addition to the current cephalometric tools available to the orthodontist that will enable better diagnosis and treatment planning in future.

\section{CONCLUSION}

A new angle; the SAR angle was developed as a diagnostic aid to assess the sagittal jaw discrepancy more consistently. Subjects with a SAR angle between 53 and $59^{\circ}$ have Class I skeletal pattern; a SAR angle less than $53^{\circ}$ indicate Class Il skeletal pattern and SAR angle greater than $59^{\circ}$ indicate Class III skeletal pattern. 


\section{REFERENCES}

1. Moyers RE, Bookstein FL, Guire KE. The concept of pattern in craniofacial growth. Am J Orthod 1979; 76:136-48.

2. Riedel RA. The relation of maxillary structures to cranium in malocclusion and in normal occlusion. Angle Orthod. $1952 ; 22: 142-145$.

3. Jacobson A. The 'Wits' appraisal of jaw disharmony. Am J Orthod 1975; 67:125-38.

4. Hussels W, Nanda RS. Analysis of factors affecting angle ANB. Am J Orthod 1984; 85:411-23.

5. Holdaway RA. Changes in relationship of points A and B during orthodontic treatment. Am J Orthod 1956; $42: 176-93$.

6. Taylor CM. Changes in the relationship of nasion, point A and point B, and the effect upon ANB. Am J Orthod 1969; $56: 143-63$.

7. Jarvinen S. An analysis of the variation of the ANB angle: a statistical appraisal. Am J Orthod 1985; 87:144-6.

8. Haynes S, Chau M. The reproducibility and repeatability of the Wits analysis. Am J Orthod Dentofac Orthop. 1995; 107:640-7.

9. Demisch A, Gebaver U, Zila W. Comparison of three cephalometric measurements of sagittal jaw relationship-angle ANB, 'Wits' appraisal and AB-occlusal angle. Trans Eur Orthod Soc 1977:269-81.

10. Rushton R, Cohen AM, Linney FD. The relationship and reproducibility of angle ANB and the 'Wits' appraisal. Br J Orthod 1991; 18:225-31.

11. Baik C Y, Ververidou M. A new approach of assessing sagittal discrepancies: the Beta angle. Am J Orthod Dentofac Orthop. 2004;126: 100-105.

12. Arvysts M G. Nonextraction treatment of severe Class II Division 2 malocclusion: part 1. Am J Orthod Dentofac Orthop. 1990; 97 : 510-521.

13. Nanda R. Biomechanics and esthetic strategies in clinical orthodontics. Elsevier, St. Louis. 2005; pp. 38-73.

14. Adenwalla S T, Kronman J H, Attarzadeh F. Porion and condyle as cephalometric landmarks: an error study. Am J Orthod Dentofac Orthop. 1988; 94: 411-415.

15. Moore R N, DuBois L M, Boice P A, Igel K A. The accuracy of measuring condylion location. Am J Orthod Dentofac Orthop. 1989; 95: 344-347.

16. Neela P K, Mascarenhas R, Husain A. A new sagittal dysplasia indicator: the Yen angle. World Jour Orthod. 2009; 10: 147-151.

17. Bhad W, Nayak S, Doshi U. A new approach of assessing sagittal dysplasia: the W angle. Eur J Orthod. $2011 ; 1-5$.

18. Bjork A. Facial growth in man, studied with the aid of metallic implants. Acta Odont Scan. 1955; 13:9-34.

19. Bjork A. Cranial base development: A follow- up X-Ray study of the individual variation in growth occurring between the ages of 12 and 20 years and its relation to cranial base and face development. Am J Orthod 1955; 41:198-225.

20. Melsen B. The cranial base: the postnatal development of the cranial base studied histologically on human autopsy material. Acta Odont Scan. 1974; 32: Suppl.64.

21. Braun S, Kittleson R, Kim K .The G-Axis: a growth vector for the mandible. Angle Orthod. 2004 ;74: 328-331.

22. Goel S, Bansal M, Kalra A. A Preliminary assessment of orthodontic superimposition. Eur J Orthod. 2004;26:217-222.

23. Viazis A D. The cranial base triangle. Jour Clinical Orthod. 1991;25:565-570,

24. Buschang P H, LaPalme L, Tanguay R, Demirijian A. The technical reliability of superimposition on cranial base and mandibular structures. Eur J Orthod. 1986:8:152-156.

25. Arat M, Koklu A, Ozdiler E, Rubenduz M, Erdogan B. Craniofacial growth and skeletal maturation: a mixed longitudinal study. Eur J Orthod. $2001 ; 23: 355-361$.

26. Torlakovic L, Faerovig E. Age-related changes of the soft tissue profile from the second to the fourth decades of life. Angle Orthod. 201 1;81:50-57. 